\title{
Finite-Frequency Observer-Based Fault Estimation and Fault-Tolerant Control for Wind Turbine
}

\author{
Ayoub EL BAKRI and Ismail Boumhidi \\ Department of physics, LISAC Laboratory, University of sidi Mohamed Ben Abdellah \\ Faculty of Sciences Dhar Mehraz BP 1796 Atlas \\ Fez, Morocco \\ ayoub.elbakri@usmba.ac.ma.
}

\begin{abstract}
This paper deals with the design problem of a robust fault estimation (FE)/ faulttolerant control (FTC) scheme for wind turbines (WT) in the finite frequency domain (FF). First, an adaptive fuzzy $\mathrm{H} \infty$ observer is constructed to simultaneously reconstruct the WT states and faults within the FF range. Throughout the observer analysis, it is only assumed that faults, uncertainties, and disturbances are bounded, which coincide with the practical requirements. In the second stage, based on the observer information, an active FTC method is proposed to stabilize the faulty WT. The controller and observer gain matrices are extracted employing the Lyapunov theory, where constraints are expressed as a set of linear matrix inequalities (LMIs). The Simulation results illustrate the best performances of the suggested strategy.
\end{abstract}

Keywords: Linear Matrix Inequalities; Fault estimation; Fault-tolerant control; Finite Frequency, T-S modelling; Wind Turbines.

\section{Introduction}

Today, wind energy and other renewable technologies, such as photovoltaic energy, are needed to meet the growing global energy demand profitably. A large number of wind energy production systems have been widely installed (onshore and offshore) as a complement to other traditional forms of power generation. Therefore, it is essential to reduce the cost of wind energy to compete or even replace a significant number of traditional power resources. One of the most critical aspects of achieving a cost-effective wind energy exploitation is to reduce the WT maintenance cost. Like any other industrial system, with external disturbances, the wind turbine plant can have undesirable behavior because of actuator or sensor faults, resulting in a higher production cost. To prevent severe imposing damages to the system and to secure a particular degree of safety, reliability, and performance. The design of robust fault estimation plays an essential task in the reliability of the WT.

However, the high nonlinearity of the wind turbine system and the wind's fluctuation make the design of the FE/FTC system a difficult task to achieve.

In the past decade, many articles have been published on fault detection, isolation, and FTC [1-8]. In particular, the process of fault estimation performs an active role in the reliability of the system. Because, based on it, a decision can be made on how and when to change the control system. One of the most potent methods used for fault estimation is the socalled observer-based approaches. The central idea is to correctly estimate faults so that a reconfigurable controller is tuned to eliminate the fault effect. For example, in [9], an adaptive observer is designed to estimate faults of the wind turbine process based on a parametervarying representation of the WT plant. In [10], the fault estimation is achieved using an augmented observer where both genetic algorithm optimization and eigenstructure assignment employed to enhance the reconstruction accuracy. In [11], the authors constructed a fault detection system based on the unscented Kalman filter (UKF) to handle faults in the generator part of the wind turbine. In [12], the authors tackle the problem of fault estimation

Received: December $19^{\text {th }}, 2019$. Accepted: August $10^{\text {th }}, 2020$

DOI: $10.15676 /$ ijeei.2020.12.3.9 
using both reduced and full-order unknown input observer (UIO). Then, the obtained reconstruct of fault is used in the FTC system's design. In [13], an adaptive sliding mode estimation method has also addressed to estimate the pitch actuator faults where an FTC scheme based on baseline PI controller was developed. In [14], an adaptive observer scheme has been employed for the FE/FTC task of a class system described by the Takagi-Sugeno descriptor systems subject to both actuator and sensor faults. It should point out that the design of a robust FE/FTC can be ensured by using the techniques mentioned above. However, these studies consider faults belong to the entire frequency range, which introduces much conservatism due to the overdesign. In practical requirements, with the fact that faults are usually located in limited frequency intervals, the generalized Kalman-Yakubovich-Popov (GKYP) lemma [15] provides a practical approach for analysis and design in FF domain. Although a couple of results exist on the FE in the finite-frequency field [16-20], none of these studies consider the robustness of the closed-loop WT system. Moreover, from a practical perspective, various factors should be considered in the design of the WT FE/FTC scheme, such as model uncertainties, estimation errors, and external disturbances.

Motivated by the above observations, in the current study, a new adaptive observer is proposed with finite-frequency specification to simultaneously reconstruct state and faults of the wind turbine systems. Since GKYP lemma cannot be used to design an observer with an adaptive mechanism, an improved method is used to derive observer constraint in terms of LMIs. After that, using the obtained estimations, an active FTC design is formed to preserve the stability of the closed-loop faulty Wind Turbine. The adaptive FF observer and the FTC are designed separately from each other. The $\mathrm{H} \infty$ optimization technique in $\mathrm{FF}$, which is more effective in addressing significant uncertainties, is used to compute the gain matrices.

The remainder of this paper is arranged as follows: Wind turbine modeling, including faults and disturbances, is presented in section 2. The FF fault estimation and fault-tolerant control design conditions and analysis are addressed in section 3 . The effectiveness of the introduced FE/FTC approach is illustrated through simulation results in section 4. Finally, section 5 concludes this article.

\section{Wind Turbine modeling}

In general, the wind turbine system is an interaction of three subsystems, respectively, known as the aerodynamics of the blades. It describes the transformation of the wind energy into forces operating on the blades. The mechanical model transmits the aerodynamic torque from the low-speed shaft to the generator by mean of the drive-train. The generator unit that describes the conversion from the mechanical power into electrical power. The mathematical equations governing the wind turbine dynamics given as follows:

$$
\begin{aligned}
& \dot{\theta}_{\Delta}=\omega_{r}-\frac{\omega_{g}}{n_{g}} \\
& \dot{\omega}_{r}=-\frac{\left(B_{d t}+B_{r}\right)}{J_{r}} \omega_{r}+\frac{B_{d t}}{n_{g} J_{r}} \omega_{g}-\frac{K_{d t}}{J_{r}} \theta_{\Delta}+\frac{T_{a}}{J_{r}} \\
& \dot{\omega}_{g}=\frac{B_{d t}}{n_{g} J_{g}} \omega_{r}-\left(\frac{B_{d t}}{n_{g}^{2} J_{g}} \omega_{g}+\frac{B_{g}}{J_{g}}\right)+\frac{K_{d t}}{n_{g} J_{g}} \theta_{\Delta}-\frac{T_{g}}{J_{g}} \\
& \ddot{\beta}=-2 \zeta \omega_{n} \dot{\beta}-\omega_{n}^{2} \beta+\omega_{n}^{2} \beta_{r} \\
& \dot{T}_{g}=-\frac{1}{\tau_{g}} T_{g}+\frac{1}{\tau_{g}} T_{g, r}
\end{aligned}
$$

where $\omega_{\mathrm{r}}[\mathrm{rad} / \mathrm{s}]$ denotes the rotational speed of the rotor, $\omega_{\mathrm{g}}[\mathrm{rad} / \mathrm{s}]$ refers to the rotational speed of the generator, and $\theta_{\Delta}$ is the torsion angle. $\mathrm{T}_{\mathrm{g}}, \mathrm{T}_{\mathrm{g}, \mathrm{r}}$ and $\tau_{\mathrm{g}}$ are the generator torque, desired input and time delay constant. $\beta$ and $\beta_{\mathrm{r}}$ are measured, and the demanded pitch angle. $\mathrm{T}_{\mathrm{a}}$ is the aerodynamic torque operating on the rotor calculated as follows. 


$$
T_{a}=\frac{1}{2} \rho \pi R^{3} C_{Q}(\lambda, \beta) v^{2}
$$

with $\rho\left[\mathrm{Kg} / \mathrm{m}^{3}\right]$ represent the density of the air, $\mathrm{R}[\mathrm{m}]$ denotes the radius of the blades, $v[\mathrm{~m} / \mathrm{s}]$ is the measured wind speed. $\mathrm{Cq}(\lambda, \beta)$ is the torque coefficient depending on both the measured angle $\beta$ and the tip speed ratio $\lambda$.

\section{A. T-S fuzzy modelling of Wind turbine}

From (1)-(5) and (6), the entire WT model can be obtained by combining its subsystems. However, because of high nonlinear behavior of the turbine aerodynamics, the proposed observer and controller's design steps cannot be directly applied. For this reason, taking advantage of the T-S modeling flexibility the aerodynamic torque is approximated as follows:

$$
T_{a} \cong T_{a, \omega r}(\theta) \omega_{r}+T_{a, \beta}(\theta) \beta+T_{a, v}(\theta) v
$$

where the partial derivative $T_{a, \beta}(\theta)=\left.\frac{\partial T_{a}}{\partial \beta}\right|_{\bar{\omega}_{r}, \bar{v}, \bar{\beta}}, T_{a, v}(\theta)=\left.\frac{\partial T_{a}}{\partial v}\right|_{\bar{\omega}_{r}, \bar{v}, \bar{\beta}}$ and $T_{a, \omega r}(\theta)=\left.\frac{\partial T_{a}}{\partial \omega_{r}}\right|_{\bar{\omega}_{r}, \bar{v}, \bar{\beta}}$ are calculated at different operating points determined by the control strategy. Then, incorporating (7) to (1) the dynamic model of the WT is written as

$$
\left\{\begin{array}{l}
\dot{x}(t)=A(\theta) x(t)+B u(t)+B_{v}(\theta) v(t) \\
y(t)=C x(t)
\end{array}\right.
$$

where $\theta=\left[\omega_{\mathrm{r}}, \beta, v\right]$ represents the vector of premise variables. The matrices of the entire WT model are:

$$
\begin{aligned}
& A(\theta)=\left[\begin{array}{cccccc}
\frac{1}{\tau_{g}} & 0 & 0 & 0 & 0 & 0 \\
0 & 0 & I & 0 & 0 & 0 \\
0 & -\omega_{n}^{2} I & -2 \xi \omega_{n} I & 0 & 0 & 0 \\
0 & \frac{1}{J_{r}} \frac{\partial T_{a}}{\partial \beta} & 0 & \frac{1}{J_{r}} \frac{\partial T_{a}}{\partial \omega_{r}}-\frac{\left(B_{d t}+B_{r}\right)}{J_{r}} & \frac{B_{d t}}{n_{g} J_{r}} & \frac{K_{d t}}{J_{r}} \\
-\frac{1}{J_{g}} & 0 & 0 & \frac{B_{d t}}{n_{g} J_{g}} & -\frac{\left(B_{d t}+n_{g} B_{g}\right)}{n_{g}^{2} J_{g}} & \frac{K_{d t}}{n_{g} J_{g}} \\
0 & 0 & 0 & 1 & -\frac{1}{n_{g}} & 0
\end{array}\right], \\
& u=\left[\begin{array}{c}
T_{g, r} \\
\beta_{r}
\end{array}\right], B=\left[\begin{array}{cc}
\frac{1}{\tau_{g}} & 0 \\
0 & 0 \\
0 & \omega_{n}^{2} I \\
0 & 0 \\
0 & 0 \\
0 & 0
\end{array}\right], B_{v}(\theta)=\left[\begin{array}{c}
0 \\
0 \\
0 \\
\frac{T_{a, v}(\theta)}{J_{r}} \\
0 \\
0
\end{array}\right], x=\left[\begin{array}{c}
T_{g} \\
\beta \\
\dot{\beta} \\
\omega_{r} \\
\omega_{g} \\
\theta_{\Delta}
\end{array}\right] \text { and } C=\left[\begin{array}{cccccc}
1 & 0 & 0 & 0 & 0 & 0 \\
0 & 1 & 0 & 0 & 0 & 0 \\
0 & 0 & 0 & 1 & 0 & 0 \\
0 & 0 & 0 & 0 & 1 & 0
\end{array}\right] \text {. }
\end{aligned}
$$

According to the wind turbine operating zones [21], concerning region 2, the controller aims to extract the maximum amount of energy from the captured wind energy. To this end, the pitch angle is set to $\beta=0$, and the power coefficient $C_{p}=\lambda C_{q}$ to $C_{p m a x}=0.45$. Consequently, the T-S representation of the WT is derived using the following IF-THEN T-S model: 


$$
\left\{\begin{array}{l}
\dot{x}(t)=\sum_{i=1}^{4} h_{i}(\theta)\left(A_{i} x(t)+B u(t)+B_{v, i} v(t)\right) \\
y(t)=\stackrel{C}{C} x(t)
\end{array}\right.
$$

with $h_{1}(\theta)=\frac{\left(\omega_{r}-\omega_{r, \text { min }}\right)}{\left(\omega_{r, \text { max }}-\omega_{r, \text { min }}\right)} \times \frac{\left(v-v_{\text {min }}\right)}{\left(v_{\text {max }}-v_{\min }\right)}, h_{2}(\theta)=\frac{\left(\omega_{r}-\omega_{r, \text { min }}\right)}{\left(\omega_{r, \text { max }}-\omega_{r, \text { min }}\right)} \times \frac{\left(v_{\text {max }}-v\right)}{\left(v_{\max }-v_{\min }\right)}$,

$$
h_{3}(\theta)=\frac{\left(\omega_{r, \text { max }}-\omega_{r}\right)}{\left(\omega_{r, \text { max }}-\omega_{r, \text { min }}\right)} \times \frac{\left(v-v_{\min }\right)}{\left(v_{\max }-v_{\min }\right)}, h_{4}(\theta)=\frac{\left(\omega_{r, \text { max }}-\omega_{r}\right)}{\left(\omega_{r, \text { max }}-\omega_{r, \text { min }}\right)} \times \frac{\left(v_{\max }-v\right)}{\left(v_{\max }-v_{\min }\right)} .
$$

B. System model with faults and disturbances.

The wind speed is the primary source of disturbances that affect the system. For this reason, the total wind turbine disturbances are modeled as $d(t)=v(t)+\zeta(t)$. Hence, by considering, the effects of faults and disturbances, the state-space model (9) of the wind turbine under fault and disturbances is rewritten as:

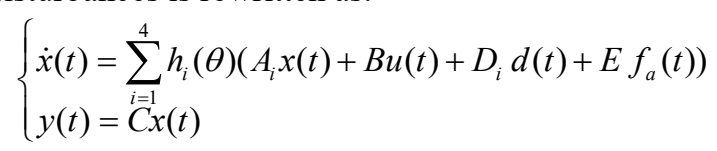

where $f_{a}(t)$ is the actuator fault vector.

\section{Adaptive fuzzy observer-based FE and FTC design}

In the following, the FE/FTC scheme design conditions are addressed in detail. Before presenting the main results, the subsequent assumptions and lemmas are considered.

Assumption 1: The faults $f_{a}$ and disturbances $d$ satisfy $\left\|f_{a}\right\| \leq \bar{f}_{a}$, and $\|d\| \leq d_{0}$. Moreover, the derivative of $f_{a}$ to time is also bounded $\left\|\dot{f}_{a}\right\| \leq f_{a \text {, max }}$.

Lemma 1 [22]. Given a scalar $\mu>0$ and a symmetric matrix $M>0$, the subsequent expression holds:

$$
2 u^{T} v \leq \frac{1}{\mu} u^{T} M u+\mu v^{T} M^{-1} v \quad u, v \in R^{n}
$$

Lemma 2 [23]. Let $U, V$ and $\Theta$ be given. There exists a matrix $\Sigma$ satisfying $\Theta+U \Sigma V^{T}+V \Sigma^{T} U<0$ if only if the next statements hold:

- $U^{\perp} \Theta U^{\perp T}<0$ and $V^{\perp} \Theta V^{\perp T}<0$.

where, $U U^{\perp}=0$ and $V V^{\perp}=0$.

Lemma 3 [15] Given the following system

$$
\left\{\begin{array}{l}
\dot{e}(t)=A_{h} e(t)+B_{h} u(t) \\
y(t)=C_{h} e(t)
\end{array}\right.
$$

For a given positive scalar $\gamma$, if the Hermitian matrices $P=P^{T}$ and $Q=Q^{T}>0$ exist so that

$$
\left(\begin{array}{cc}
A_{h} & B_{h} \\
I & 0
\end{array}\right)^{T} \Xi_{Q}\left(\begin{array}{cc}
A_{h} & B_{h} \\
I & 0
\end{array}\right)+\left(\begin{array}{cc}
C_{h} & 0 \\
0 & I
\end{array}\right)^{T} \underbrace{\left(\begin{array}{cc}
I & 0 \\
0 & -\gamma^{2} I
\end{array}\right)}_{\Pi}\left(\begin{array}{cc}
C_{h} & 0 \\
0 & I
\end{array}\right)<0
$$

with the matrix $\Xi_{Q}$ as given in Table 1. Therefore, the error system (15) is considered asymptotically stable with the prescribed $\mathrm{H} \infty$ performance index in FF range. 
Table 1. $\Xi_{Q}$ for all frequency ranges

\begin{tabular}{|c|c|c|}
\hline $\begin{array}{c}\text { Low-frequency range } \\
|\omega| \leq \omega_{l}\end{array}$ & $\begin{array}{c}\text { Middle-frequency range } \\
\omega_{1} \leq \omega \leq \omega_{2}, \omega_{0}=\left(\omega_{1}+\omega_{2}\right) / 2\end{array}$ & $\begin{array}{c}\text { High-frequency range } \\
|\omega| \geq \omega_{h}\end{array}$ \\
\hline$\Xi_{Q}=\left(\begin{array}{cc}-Q & P \\
P & \omega_{l}^{2} Q\end{array}\right)$ & $\Xi_{Q}=\left(\begin{array}{cc}-Q & P+j \omega_{0} Q \\
P-j \omega_{0} Q & -\omega_{1} \omega_{2} Q\end{array}\right)$ & $\Xi_{Q}=\left(\begin{array}{cc}Q & P \\
P & -\omega_{h}^{2} Q\end{array}\right)$ \\
\hline
\end{tabular}

\section{A. FF observer-based fault estimation}

Given the wind turbine model in (10), the next FF adaptive observer (FFAO) shape is considered [24]:

$$
\left\{\begin{array}{c}
\dot{\hat{x}}(t)=\sum_{i=1}^{4} h_{i}(\theta)\left(A_{i} x(t)+B u(t)+E \hat{f}_{a}(t)-L_{i}(y(t)-\hat{y}(t))\right) \\
\hat{y}(t)=C \hat{x}(t) \\
\dot{\hat{f}}_{a}(t)=\Gamma F\left(\dot{e}_{y}(t)+\sigma e_{y}(t)\right)
\end{array}\right.
$$

where $\hat{x}(t), \hat{y}(t)$ are the estimate of the wind turbine state and output vectors, respectively. $\hat{f}_{a}(t)$ is the estimation of the fault and $e_{y}(t)$ is the output reconstruction error. the scalar $\sigma>0$, and the matrix $\Gamma=\Gamma^{T}>0 \Gamma$ are tuning parameters, and $F$ and $L_{i}$ are the gain matrices to be solved. Let defines the state, output, and fault reconstruction error vectors as:

$$
e_{x}(t)=x(t)-\hat{x}(t), e_{y}(t)=y(t)-\hat{y}(t) \text { and } e_{f}(t)=f_{a}(t)-\hat{f}_{a}(t) .
$$

Based on (10) and (14) we have the estimating error system as follows

$$
\left\{\begin{array}{l}
\dot{e}_{x}(t)=\sum_{i=1}^{4} h_{i}(\theta)\left(\left(A_{i}-L_{i} C\right) e_{x}(t)+E e_{f}(t)+D_{i} d(t)\right) \\
e_{y}(t)=C e_{x}(t)
\end{array}\right.
$$

Thus, the observer design can be formulated as follows:

Given the wind turbine TS fuzzy representation in (10), determine a fuzzy adaptive observer in the form of (14) so that the next constraints hold:

- For $d(t)=0$ and $f_{a}(t)=0$ the error system (15) is asymptotically stable.

- For $d(t)=0$ and $f_{a}(t) \neq 0$, the Ho performance from $e_{f}(t)$ to $e_{y}(t)$ is less than a given scalar $\gamma_{f}>0$ in low frequency range.

- For $d(t) \neq 0$ and $f_{a}(t)=0$, the $H \infty$ performance from $d(t)$ to $e_{y}(t)$ is less than a given scalar $\gamma_{d}>0$.

For the sake of clarity, the following notations defined.

$$
A_{h}=\sum_{i=1}^{4} h_{i}(\theta) A_{i}, D_{h}=\sum_{i=1}^{4} h_{i}(\theta) D_{i}, L_{h}=\sum_{i=1}^{4} h_{i}(\theta) L_{i} .
$$

Theorem 1 (FE). For a fixed scalars $\sigma>0, \mu>0, \gamma_{f}>0$ and $\gamma_{d}>0$, the error system (15) is called asymptotically stable and the Ho performances indices $\gamma_{f}$ and $\gamma_{d}$ hold, if there is a symmetric matrices $P>0, Q>0$, and $X>0$, general matrices $Y_{h}, \bar{P}$ and $G$ which satisfy:

$$
\begin{gathered}
E^{T} X=F C \\
\left(\begin{array}{cc}
-X-X^{T} & P+X A_{h}-Y_{h} C-X^{T} \\
* & \left(X A_{h}-Y_{h} C\right)+\left(X A_{h}-Y_{h} C\right)^{T}
\end{array}\right)<0
\end{gathered}
$$




$$
\begin{aligned}
& \left(\begin{array}{cccc}
-Q-X-X^{T} & \bar{P}+\left(X A_{h}-Y_{h} C\right)-X^{T} & X E & 0 \\
* & \omega_{l}^{2} Q+\left(X A_{h}-Y_{h} C\right)+\left(X A_{h}-Y_{h} C\right)^{T} & X E-\frac{1}{\sigma}\left(X A_{h}-Y_{h} C\right)^{T} E & C^{T} \\
* & * & -\gamma_{f}^{2} I-\frac{2}{\sigma} E^{T} X E+\frac{1}{\sigma \mu} G & 0 \\
* & * & * & -I
\end{array}\right)<0 \\
& \left(\begin{array}{ccc}
\left(X A_{h}-Y_{h} C\right)+\left(X A_{h}-Y_{h} C\right)^{T} & X D & C^{T} \\
* & -\gamma_{d}^{2} & 0 \\
* & * & -I
\end{array}\right)<0
\end{aligned}
$$

Proof. Let define a Lyapunov function as follows $V(t)=\int_{0}^{\infty} \dot{V}_{1}(t) d t+\int_{0}^{\infty} \dot{V}_{2}(t) d t$. With $\dot{V}_{1}(t)=\xi^{T}(t)\left(\Psi^{T} \Xi_{Q} \Psi+\Lambda^{T} \Pi \Lambda\right) \xi(t)$ and $\dot{V}_{2}(t)=\frac{2}{\sigma} e_{f}^{T}(t) \Gamma^{-1} \dot{e}_{f}(t)$. Based on (15), the derivative $\dot{V}_{2}$ can be proved to be equal to:

$\dot{V}_{2}(t)=\frac{2}{\sigma} e_{f}^{T}(t) \Gamma^{-1} \dot{f}_{a}(t)-\frac{2}{\sigma} e_{f}^{T}(t) F C\left(A_{h}-L_{h} C\right) e_{x}(t)-\frac{2}{\sigma} e_{f}^{T}(t) F C E e_{f}(t)-2 e_{f}^{T}(t) F C e_{x}(t)$.

From lemma 1 and the equality (16) we have $\dot{V}_{2}(t) \leq-\frac{2}{\sigma} e_{f}^{T}(t) E^{T} X\left(A_{h}-L_{h} C\right) e_{x}-\frac{2}{\sigma} e_{f}^{T}(t) E^{T} X E e_{f}(t)+\frac{1}{\sigma \mu} e_{f}^{T}(t) G e_{f}(t)+\frac{\mu}{\sigma} \dot{f}_{a}^{2}(t) \lambda_{\max }\left(\Gamma^{-1} G^{-1} \Gamma^{-1}\right)$ which is equal to $\dot{V}_{2}(t) \leq \xi^{T}(t)(\phi) \xi(t)+\chi$ where

$$
\phi=\left(\begin{array}{cc}
0 & -\frac{1}{\sigma}\left(A_{h}-L_{h} C\right)^{T} X E \\
* & -\frac{2}{\sigma} E^{T} X E+\frac{1}{\sigma \mu} G
\end{array}\right), \xi(t)=\left[\begin{array}{ll}
e_{x}^{T} & e_{f}^{T}
\end{array}\right]^{T} \text { and } \chi=f_{a, \max }^{2} \lambda_{\max }\left(\Gamma^{-1} G^{-1} \Gamma^{-1}\right) .
$$

Therefore, the overall derivative of the Lyapunov candidate function is rewritten as

$$
\begin{gathered}
\dot{V}(t) \leq \xi^{T}(t)\left(\Psi^{T} \Xi_{Q} \Psi+\Phi\right) \xi(t)+\chi \text { where } \\
\Phi=\left(\begin{array}{cc}
C^{T} C & -\frac{1}{\sigma}\left(A_{h}-L_{h} C\right)^{T} X E \\
* & -\gamma_{f}^{2} I-\frac{2}{\sigma} E^{T} X E+\frac{1}{\sigma \mu} G
\end{array}\right) .
\end{gathered}
$$

Now, let define $\eta=\left(\begin{array}{cc}\Xi_{Q} & 0 \\ 0 & \Phi\end{array}\right)$ then $\left(\Psi^{T} \Xi_{Q} \Psi+\Phi\right)$ can be formulated as

$$
\left(\begin{array}{cc}
A_{h}-L_{h} C & E \\
I & 0 \\
0 & I
\end{array}\right)^{T}\left\{\left(\begin{array}{ccc}
I & 0 & 0 \\
0 & I & 0 \\
\hline 0 & I & 0 \\
0 & 0 & I
\end{array}\right)^{T} \eta\left(\begin{array}{ccc}
I & 0 & 0 \\
0 & I & 0 \\
\hline 0 & I & 0 \\
0 & 0 & I
\end{array}\right)\right\}\left(\begin{array}{cc}
A_{h}-L_{h} C & E \\
I & 0 \\
0 & I
\end{array}\right)<0
$$

which has the same form of $U^{\perp} \Theta U^{\perp T}<0$ with 


$$
\Theta=\left(\begin{array}{ccc}
-Q & \bar{P} & 0 \\
* & \omega_{l}^{2} Q+C^{T} C & -\frac{1}{\sigma}\left(A_{h}-L_{h} C\right)^{T} X E \\
* & * & -\gamma_{f}^{2} I-\frac{2}{\sigma} E^{T} X E+\frac{1}{\sigma \mu} G
\end{array}\right)
$$

Thus, based on the projection lemma (20) is equivalent to

$$
\Delta=\Theta+\left(\begin{array}{c}
-I \\
\left(A_{h}-L_{h} C\right)^{T} \\
E^{T}
\end{array}\right) X\left(\begin{array}{lll}
I & I & 0
\end{array}\right)+\left(\begin{array}{lll}
I & I & 0
\end{array}\right)^{T} X\left(\begin{array}{c}
-I \\
\left(A_{h}-L_{h} C\right)^{T} \\
E^{T}
\end{array}\right)^{T}<0
$$

Which is equal to (18) by Schur complement with $Y_{h}=X L_{h}$. Therefore, when $(\Delta)<0$ one can find $\dot{V}(t)<-\lambda_{\min }(\Delta)\|\xi(t)\|^{2}+\chi$. It means that $\dot{V}<0$ for $\lambda_{\min }(\Delta)\|\xi(t)\|^{2}>\chi$ and that the state and fault estimation error will be bounded and converge to a small set.

To ensure $\left(A_{h}-L_{h} C\right)$ is asymptotically stable the following Lyapunov candidate function is introduced

$$
\bar{V}=\left(A_{h}-L_{h} C\right)^{T} P+P\left(A_{h}-L_{h} C\right)<0
$$

Which can be rewritten in the form of

$$
\left(\begin{array}{c}
\left(A_{h}-L_{h} C\right) \\
I
\end{array}\right)^{T}\left(\begin{array}{ll}
0 & P \\
P & 0
\end{array}\right)\left(\begin{array}{c}
\left(A_{h}-L_{h} C\right) \\
I
\end{array}\right)<0
$$

Defining $\Theta=\left(\begin{array}{cc}0 & P \\ P & 0\end{array}\right), V=\left(\begin{array}{c}-I \\ \left(A_{h}-L_{h} C\right)^{T}\end{array}\right)^{T}$ and $\Sigma=\left(\begin{array}{c}X \\ X\end{array}\right)$. Thus, by the projection lemma we get.

$$
\left(\begin{array}{ll}
0 & P \\
P & 0
\end{array}\right)+\left(\begin{array}{c}
X \\
X
\end{array}\right)\left(\begin{array}{ll}
-I & A_{h}-L_{h} C
\end{array}\right)+\left(\begin{array}{ll}
-I & A_{h}-L_{h} C
\end{array}\right)^{T}\left(\begin{array}{c}
X \\
X
\end{array}\right)^{T}<0
$$

which gives the inequality (17).

\section{B. Finite Frequency-based FTC scheme}

In low operating mode, the wind turbine runs with the pitch angle $\beta=0$ while tracking the desired power reference. In the high operating mode, the pitch controller becomes effective in regulating the current and restricting the rotational speed of the generator at maximum value. Thus, it sets the power of the turbine at the desired value. As a result, the wind turbine system operates at a variable frequency [25]. In this particular, a limited frequency range ([$\omega_{1}$ $\left.\omega_{2}\right] \mathrm{Hz}$ ) can cover the complete operational bounds of the WT. To this purpose, the controller design conditions will be computed in the middle-frequency range to further reduce the conservatism. Figure 1 describes the structure of the FF-FTC scheme.

Let consider the following FTC law:

$$
u(t)=K_{h} \hat{x}(t)-\hat{f}_{a}(t)
$$

Then, the closed loop system is written as

$$
\dot{x}(t)=\left(A_{h}+B K_{h}\right) x(t)-B K_{h} e_{x}(t)+E e_{f}(t)+D_{h} d(t)
$$




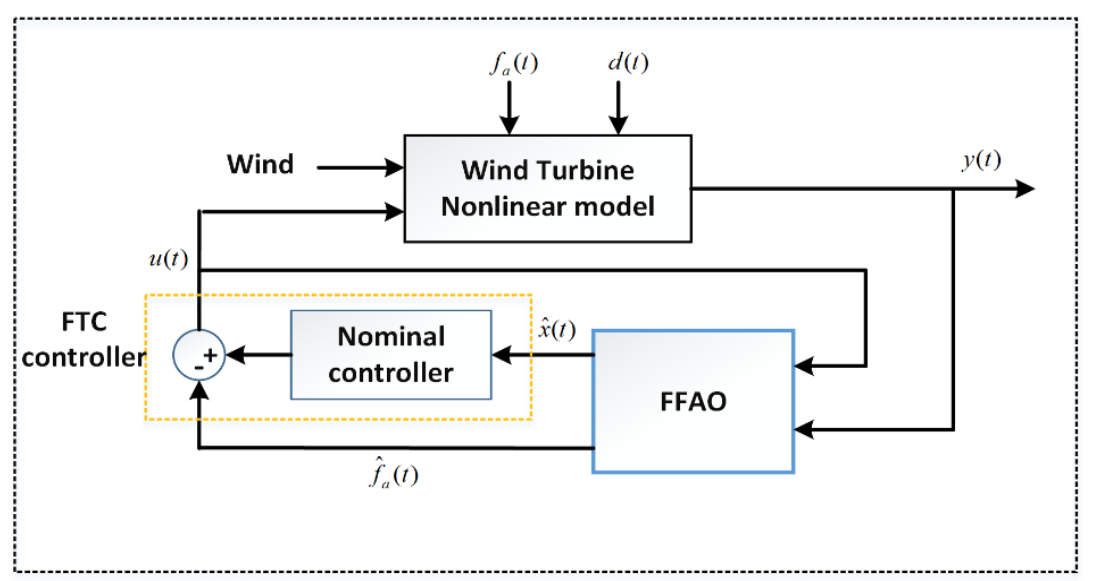

Figure.1 The finite-frequency observer-based FTC scheme.

Let $\bar{x}(t)=\left[\begin{array}{ll}x^{T}(t) & e_{x}^{T}(t)\end{array}\right]^{T}, \Upsilon(t)=\left[\begin{array}{ll}e_{f}^{T}(t) & d^{T}(t)\end{array}\right]^{T}$. The following augmented structure hold:

$$
\begin{aligned}
& \left\{\begin{array}{l}
\dot{\bar{x}}(t)=\bar{A}_{h}^{c} \bar{x}(t)+W_{h} \Upsilon(t) \\
y(t)=\bar{C} \bar{x}(t)
\end{array}\right. \\
& \text { where } \bar{A}_{h}=\left(\begin{array}{cc}
A_{h} & 0 \\
0 & A_{h}-L_{h} C
\end{array}\right), \bar{E}=\left(\begin{array}{l}
E \\
E
\end{array}\right), \bar{D}_{h}=\left(\begin{array}{c}
D_{h} \\
D_{h}
\end{array}\right), W_{h}=\left[\begin{array}{ll}
\bar{E} & \bar{D}_{h}
\end{array}\right] \bar{K}_{h}=\left(\begin{array}{ll}
K_{h} & -K_{h}
\end{array}\right), \\
& \bar{C}=\left(\begin{array}{ll}
C & 0
\end{array}\right), \bar{B}=\left(\begin{array}{l}
B \\
0
\end{array}\right) \text { and } \bar{A}_{h}^{c}=\bar{A}_{h}+\bar{B} \bar{K}_{h} .
\end{aligned}
$$

Theorem 2 (FTC): Given a scalar $\gamma>0$, the closed-loop system (27) is asymptotically stable with $\mathrm{H} \infty$ performance index $\gamma$ in the middle frequency, if there is Hermitian matrices $P=\left(\begin{array}{cc}P_{1} & P_{2} \\ * & P_{3}\end{array}\right), Q=\left(\begin{array}{cc}Q_{1} & Q_{2} \\ * & Q_{3}\end{array}\right)>0$ and $X=\left(\begin{array}{cc}X_{1} & 0 \\ 0 & X_{1}\end{array}\right)$, and matrices $\bar{Y}_{h}=\left[\begin{array}{ll}Y_{h} & -Y_{h}\end{array}\right]$ so that the next condition holds:

$$
\left(\begin{array}{cccc}
-Q-X-X^{T} & P+j \omega_{0} Q-X+\bar{A}_{h}^{c} X & W_{h} & 0 \\
* & -\omega_{1} \omega_{2} Q+\left(\bar{A}_{h} X+\bar{B} \bar{Y}_{h}\right)+\left(\bar{A}_{h} X+\bar{B} \bar{Y}_{h}\right)^{T} & W_{h} & X \bar{C} \\
* & * & -\gamma^{2} I & 0 \\
* & * & * & -I
\end{array}\right)<0
$$

Proof: Let $\Xi_{Q}=\left(\begin{array}{cc}-\bar{Q} & \bar{P}+j \omega_{0} \bar{Q} \\ \bar{P}-j \omega_{0} \bar{Q} & -\omega_{1} \omega_{2} \bar{Q}\end{array}\right)$ and $\Phi=\left(\begin{array}{cc}\bar{C}^{T} \bar{C} & 0 \\ 0 & -\gamma^{2} I\end{array}\right)$, thus, by mean of the lemma 3 we have $\Psi^{T} \Xi_{Q} \Psi+\Phi<0$. Which is equivalent to

$$
U^{\perp} \Theta U^{\perp T}<0
$$

with $\Theta=\left(\begin{array}{ccc}-\bar{Q} & \bar{P}+j \omega_{0} \bar{Q} & 0 \\ \bar{P}-j \omega_{0} \bar{Q} & -\omega_{1} \omega_{2} \bar{Q}+\bar{C}^{T} \bar{C} & 0 \\ 0 & 0 & -\gamma^{2} I\end{array}\right)$, and $U^{\perp}=\left(\begin{array}{ll}\bar{A}_{h}^{c} & W_{h} \\ I & 0 \\ 0 & I\end{array}\right)^{T}$. Using the projection lemma (29) is equivalent to 


$$
\left(\begin{array}{ccc}
-\bar{Q}-Z-Z^{T} & \bar{P}+j \omega_{0} \bar{Q}+Z \bar{A}_{h}^{c}-Z^{T} & Z W_{h} \\
* & -\omega_{1} \omega_{2} \bar{Q}+\bar{C}^{T} \bar{C}+Z \bar{A}_{h}^{c}+\left(Z \bar{A}_{h}^{c}\right)^{T} & Z W_{h} \\
* & * & -\gamma^{2} I
\end{array}\right)<0
$$

through multiplying both sides of (30) by $J=\left\{\begin{array}{lll}Z^{-1} & Z^{-1} & I\end{array}\right\}$ and its transpose and setting $X=Z^{-1}, Q=Z^{-1} \bar{Q} Z^{-T}, P=Z^{-1} \bar{P} Z^{-T}$, the condition (30) is equal to (28).

\section{Simulation results}

The proposed finite-frequency FE/FTC scheme is tested in this section. Case studies are carried out through Matlab/Simulink, where Sudemi toolbox is used to solve the obtained LMIs constraints. The parameters of the used wind turbine correspond to parameters of the 4.8 MW WT given in [21] where the inertia of the rotor and generator $J_{r}=55 \cdot 10^{6} \mathrm{~kg} \cdot \mathrm{m}^{2}$, $J_{g}=390 \mathrm{~kg} \cdot \mathrm{m}^{2}$. The viscous friction of the rotor and the generator $B_{r}=7.11 \mathrm{Nms} / \mathrm{rad}$, $B_{g}=45.6 \mathrm{Nms} / \mathrm{rad}$, the torsion stiffness and damping $K_{d t}=2.7 \cdot 10^{9} \mathrm{Nm} / \mathrm{rad}$, $B_{d t}=775.49 \mathrm{Nms} / \mathrm{rad}, R=57 \mathrm{~m}$. During the simulation, the nonlinear wind turbine model under disturbances and actuator faults is directly used to evaluate the FF observer-based FTC scheme. The wind speed profile used in simulations consist of real wind speed sequence collected at north of morocco as shown in Figure.2.

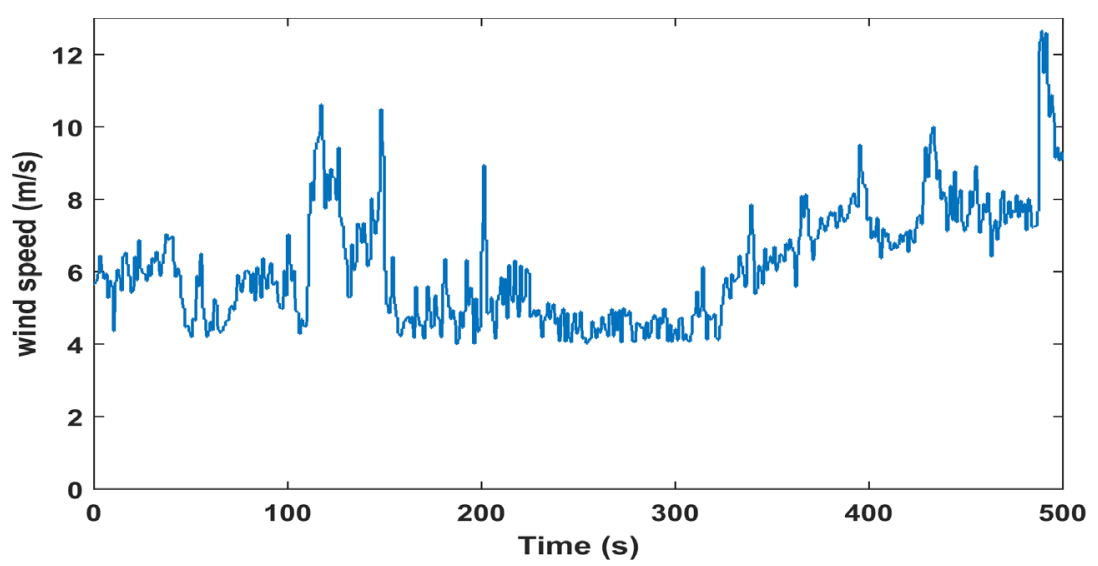

Figure 2. wind speed profile $(\mathrm{m} / \mathrm{s})$

Due to the wind turbine nonlinearity, the wind input disturbance has a critical stochastic component on the wind turbine that makes the open-loop operation becomes unstable. The eigenvalues of the open-loop wind turbine are the following.

$$
\begin{aligned}
& \lambda\left(A_{1}\right)=\left[\begin{array}{llllll}
-0.0498+28.1633 i & -0.0498-28.1633 i & 0.1483 & -6.6660+8.8880 i & -6.6660-8.8880 i & -50.0000
\end{array}\right]^{T}, \\
& \lambda\left(A_{3}\right)=\left[\begin{array}{llllll}
-0.0390+28.1630 i & -0.0390-28.1630 i & 0.4760 & -6.6660+8.8880 i & -6.6660-8.8880 i & -50.0000
\end{array}\right]^{T} \\
& \lambda\left(A_{2}\right)=\lambda\left(A_{1}\right), \quad \lambda\left(A_{4}\right)=\lambda\left(A_{3}\right) .
\end{aligned}
$$

To stabilize the WT and reach the desired control objectives. The control low given in (25) is applied to the nonlinear WT model. The closed-loop eigenvalues are the following:

$$
\begin{aligned}
& \lambda\left(A_{1}+B K_{1}\right)=\left[\begin{array}{llllll}
-31.8084 & -0.0693+28.1625 i & -0.0693-28.1625 i & -0.0616 & -6.6660+8.8880 i & -6.6660-8.8880 i
\end{array}\right]^{T} \\
& \lambda\left(A_{2}+B K_{2}\right)=\left[\begin{array}{llllll}
-35.3665 & -0.0678+28.1628 i & -0.0678-28.1628 i & -0.0615 & -6.6660+8.8880 i & -6.6660-8.8880 i
\end{array}\right]^{T}
\end{aligned}
$$




$$
\begin{aligned}
& \lambda\left(A_{3}+B K_{3}\right)=\left[\begin{array}{llllll}
-88.9144 & -0.0576+28.1704 i & -0.0576-28.1740 i & -0.0293 & -6.6660+8.8880 i & -6.6660-8.8880 i
\end{array}\right]^{T} \\
& \lambda\left(A_{4}+B K_{4}\right)=\left[\begin{array}{llllll}
-81.4394 & -0.0580+28.1696 i & -0.0580-28.1696 i & -0.0294 & -6.6660+8.8880 i & -6.6660-8.8880 i
\end{array}\right]^{T}
\end{aligned}
$$

\section{A. Actuator fault estimation}

In this subsection, two actuator faults applied to examine the capability of the proposed fault estimation observer. The chosen faults models for the actuator faults are:

$$
\begin{aligned}
& f_{a, 1}(t) \begin{cases}1+0.01^{*} \sin \left(0.1^{*} t\right) & \text { if } 100 s<t<150 s \\
0 & \text { if others }\end{cases} \\
& f_{a, 2}(t) \begin{cases}0.8+\sin \left(0.5^{*} t\right) & \text { if } 200<t<250 s \\
0 & \text { if others }\end{cases}
\end{aligned}
$$

Simulation results with comparison to the method given in [26] presented next to show the superiority of the approach. Choosing the tuning parameter values of the adaptive observer as follows: $\sigma=2, \mu=5$, results in the following eigenvalues:

$$
\begin{aligned}
& \lambda\left(A_{1}-L_{1} C\right)=\left[\begin{array}{llllll}
-135412.54 & -13.21 & -3.39 & -0.32 & -1.29 & -1.70
\end{array}\right]^{T}, \\
& \lambda\left(A_{2}-L_{2} C\right)=\lambda\left(A_{1}-L_{1} C\right), \\
& \lambda=\left(A_{3}-L_{3} C\right)=\left[\begin{array}{llllll}
-135412.60 & -13.22 & -3.38 & -0.31 & -1.68 & -1.28
\end{array}\right]^{T}, \\
& \lambda\left(A_{4}-L_{4} C\right)=\lambda\left(A_{3}-L_{3} C\right) .
\end{aligned}
$$

Figures (3), (4) and (5) and (6) display the actuator faults profiles along with the obtained estimation curves and errors.

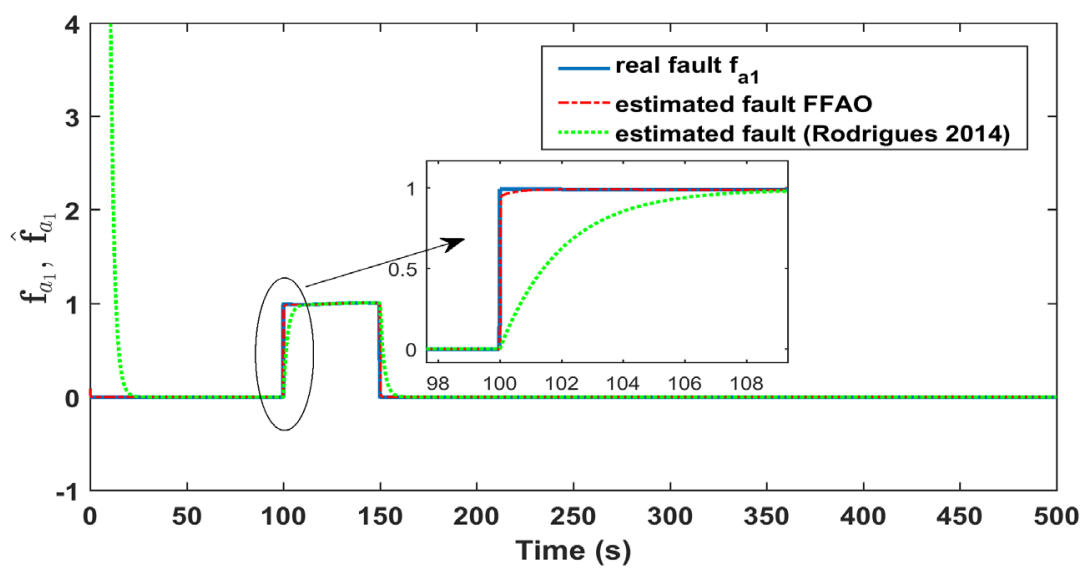

Figure 3. Estimation results of the fault $f_{a, 1}$ 


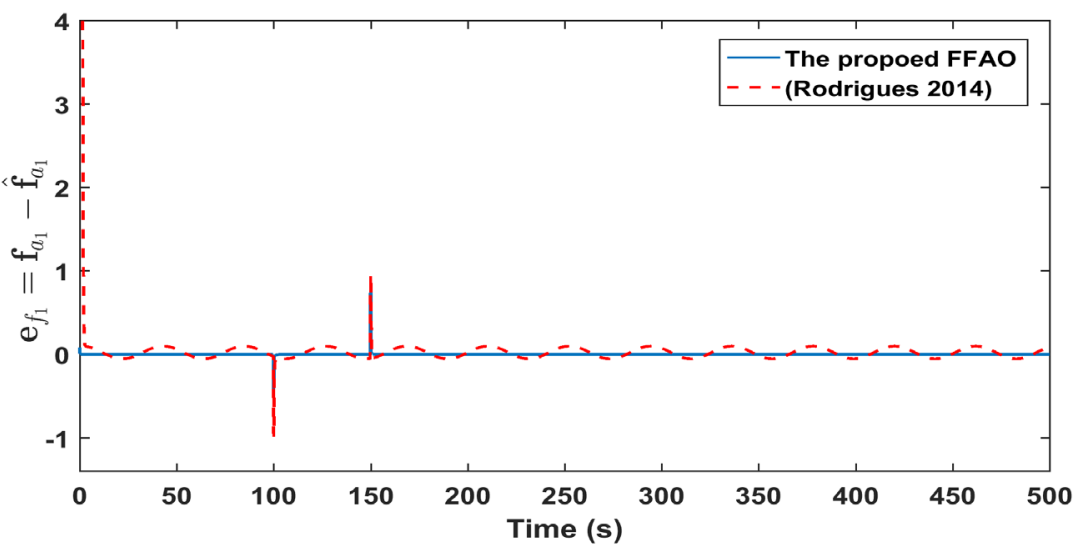

Figure 4. Estimation error of the fault $f_{a, 1}$

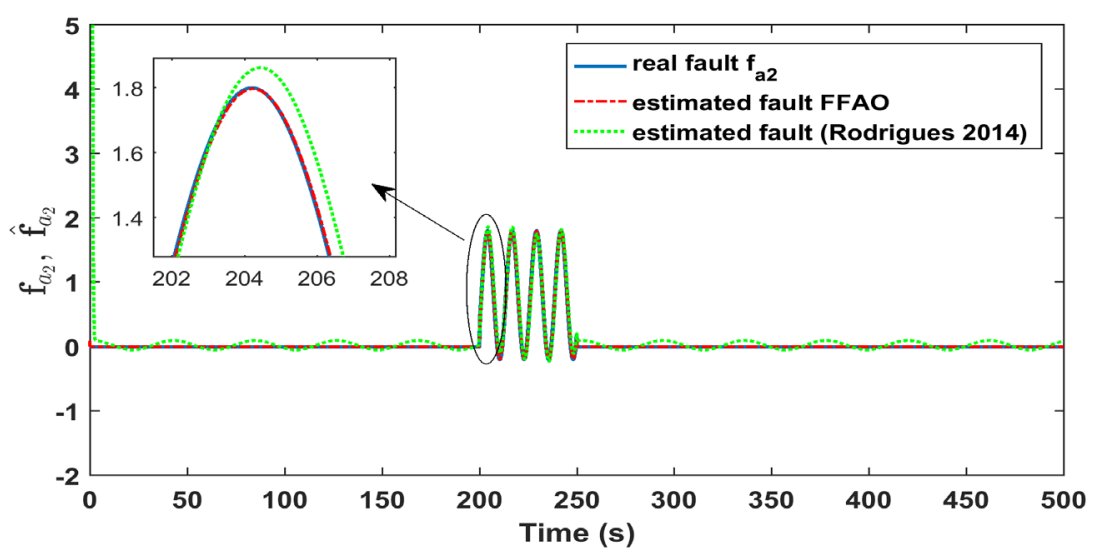

Figure 5. Fault estimation of the fault $f_{a, 2}$

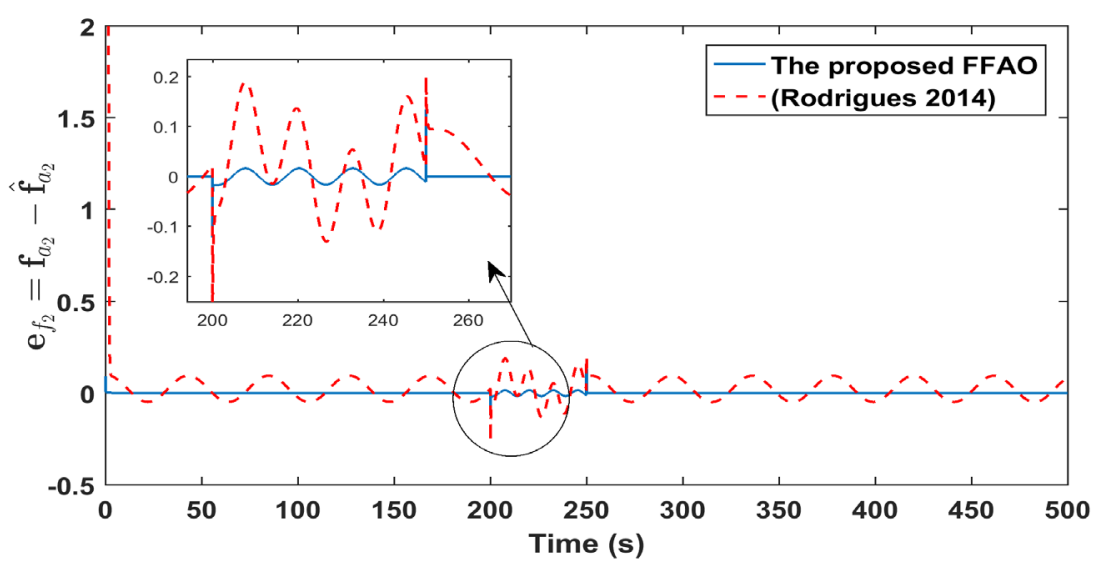

Figure 6. Estimation error of the fault $f_{a, 2}$

From those plots, one can observe that the obtained fault estimates track both constant fault and time-varying fault signals. However, the proposed FFAO exhibit robust estimation with functional disturbances attenuation of the actuator faults compared to the method given in [26], which is explained with the fast convergence speed and with reduced fluctuations toward 
significant disturbances generated by the proposed FFAO.

\section{B. Actuator fault compensation}

To check the fault tolerance capacity of the proposed FFAO method, dynamic simulation of the WT system with a sudden fault occurrence in the period [20 30s] is carried out under the wind sequence given in Figure.2.

Figures. 7-9 shows the generator speed, torque, and power response curves under the active FTC method in the presence of the fault. It worth noting that a rate limitation of $1000 \mathrm{Nm} / \mathrm{s}$ is applied to the actuator torque in simulation on account for physical system constraint.

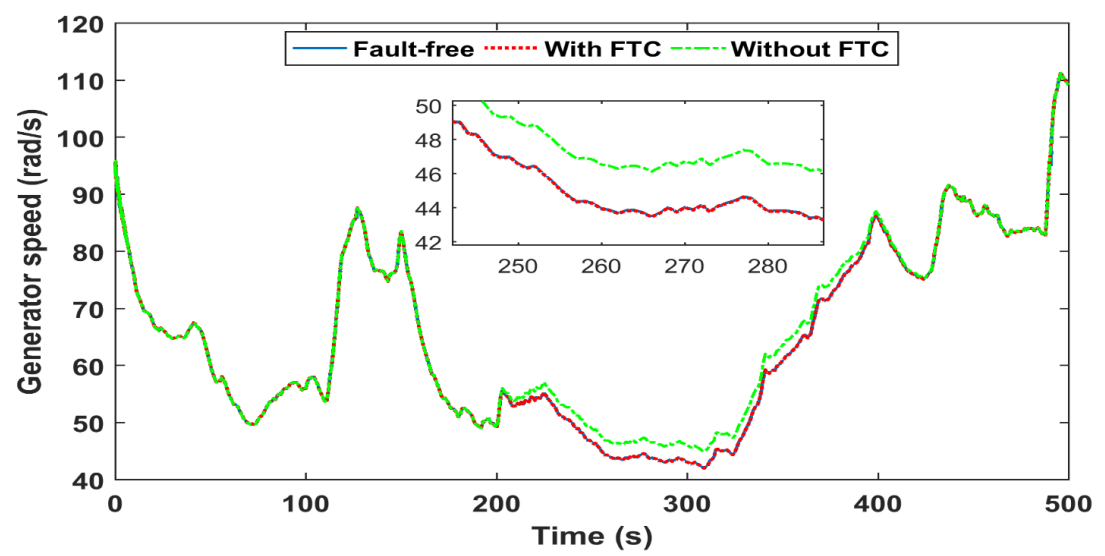

Figure 7. Generator speed $\omega_{\mathrm{g}}$ under the proposed FTC

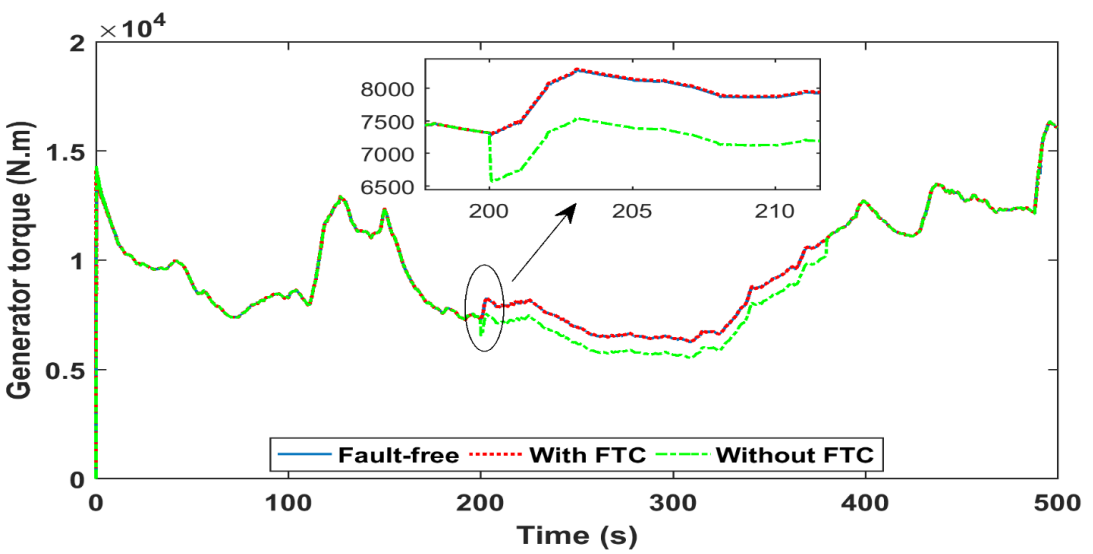

Figure 8 . The Generator torque $T_{g}$ under the proposed FTC 


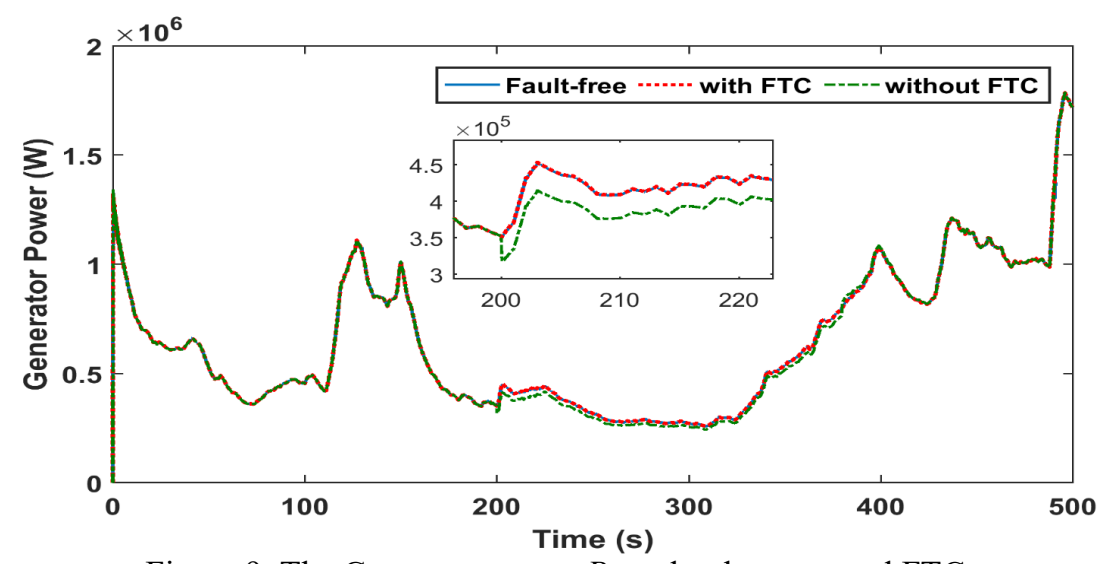

Figure 9. The Generator power $P_{g}$ under the proposed FTC

As can be seen from those curves, the presence of fault causes an undesired deviation in the rotation speed of the generator, causing a non-optimal power extraction. However, from the results, we can see that the proposed FE/TFC scheme employed in this work quickly recover the desired performance and ensure the stability of the closed-loop wind turbine regardless to the existence of disturbances and faults. Thus, leading to optimal power extraction.

\section{Conclusion}

In this study, a finite-frequency fault estimation/fault-tolerant control scheme is developed and applied for the wind turbine. Given the unpredictable actuator fault in the WT, an adaptive fuzzy observer is designed to estimate the system's state and faults simultaneously. As shown by simulation results, the chosen $\mathrm{H} \infty$ synthesis in the finite-frequency field allows to disturbances impact to be significantly mitigated, and, consequently, to the fault estimate to be very smooth and accurate. Furthermore, simulation results show that the FTC methodology presented in this study can easily sustain the stability, reliability, and dominant performances of the WT during the occurrence of actuator faults.

\section{References}

[1]. H. Habibi, I. Howard, S. Simani, "Reliability improvement of wind turbine power generation using model-based fault detection and fault tolerant control: A review", Renewable Energy, vol. 135, pp. 877-96, 2019.

[2]. Z. Hameed, Y.S. Hong, Y.M. Cho, S.H. Ahn, C.K. Song, "Condition monitoring and fault detection of wind turbines and related algorithms: A review", Renewable and Sustainable Energy Reviews, Vol. 13, pp. 1-39, 2009.

[3]. X. Wei, M. Verhaegen, T. van Engelen, "Sensor fault detection and isolation for wind turbines based on subspace identification and Kalman filter techniques", International Journal of Adaptive Control and Signal Processing, vol. 24, pp. 687-707, 2010.

[4]. M. Witczak, D. Rotondo, V. Puig, F. Nejjari, M. Pazera, "Fault estimation of wind turbines using combined adaptive and parameter estimation schemes", Int J Adapt Control Signal Process, vol. 32, pp. 549- 567, 2018.

[5]. D. Yu, Z.M. Chen, K.S. Xiahou, M.S. Li, T.Y. Ji, Q.H. Wu, "A radically data-driven method for fault detection and diagnosis in wind turbines", International Journal of Electrical Power \& Energy Systems. Vol. 99, pp. 577-84, 2018.

[6]. M.L. Corradini, G. Ippoliti, G. Orlando, "Sensorless efficient fault-tolerant control of wind turbines with geared generator", Automatica, Vol. 62, pp. 161-67, 2015.

[7]. A. El Bakri, M. Koumir, I. Boumhidi, "Extreme learning machine-based non-linear observer for fault detection and isolation of wind turbine", Australian Journal of Electrical and Electronics Engineering. Vol.16, pp. 12-20, 2019. 
[8]. H. Schulte, E. Gauterin, "Fault-tolerant control of wind turbines with hydrostatic transmission using Takagi-Sugeno and sliding mode techniques", Annual Reviews in Control. Vol. 40, pp. 82-92, 2015.

[9]. H.S. Zhiwei, G.X. Liu, K. Busawon, "Parameter-varying modelling and fault reconstruction for wind turbine systems", Renewable Energy, Vol. 116, pp. 145-52, 2018.

[10]. S. Odofin, E. Bentley, D. Aikhuele, "Robust fault estimation for wind turbine energy via hybrid systems", Renewable Energy. Vol. 120, pp. 289-99, 2018.

[11]. S. Yu, K. Emami, T. Fernando T, H.C. Herbert, K.P. Wong, "State Estimation of Doubly Fed Induction Generator Wind Turbine in Complex Power Systems", IEEE Transactions on Power Systems, Vol. 31, pp. 4935-4944, 2016.

[12]. J. Lan, R.J. Patton, "A new strategy for integration of fault estimation within faulttolerant control”, Automatica. Vol. 69, pp. 48-59, 2016.

[13]. J. Lan, R.J. Patton, X. Zhu, "Fault tolerant wind turbine pitch control using adaptive sliding mode estimation", Renewable Energy, Vol. 116, pp. 219-231, 2018.

[14]. D. Kharrat, H. Gassara, A. El Hajjaji, M. Chaabane, "Adaptive Observer and Fault Tolerant Control for Takagi-Sugeno Descriptor Nonlinear Systems with Sensor and Actuator Faults", International Journal of Control, Automation and Systems, Vol. 16, pp. 972-982, 2018.

[15]. T. Iwasaki, S. Hara, "Generalized KYP lemma: Unified frequency domain inequalities with design applications", IEEE Transactions on Automatic Control, Vol. 50, pp. 41-59, 2005.

[16]. A. El-Amrani, A. El Hajjaji, A. Hmamed, I. Boumhidi, "Finite Frequency Filter Design for T-S Fuzzy Continuous Systems", 2018 IEEE International Conference on Fuzzy Systems, Rio de Janeiro, July 8-13, 2018.

[17]. X-J. Li, G-H Yang, "Adaptive $\mathrm{H} \infty$ control in finite frequency domain for uncertain linear systems”, Information Sciences. Vol. 314, pp. 14-27, 2015.

[18]. A. Chibani, M. Chadli, P. Shi, N.B. Braiek "Fuzzy Fault Detection Filter Design for TS Fuzzy Systems in the Finite-Frequency Domain", IEEE Transactions on Fuzzy Systems Vol. 25, pp. 1051-1061, 2017.

[19]. D. Du, S. Xu, V. Cocquempot, "Actuator fault estimation for discrete-time switched systems with finite-frequency", Systems \& Control Letters, Vol.108, pp. 64-70, 2017.

[20]. Z. Wang, P. Shi, C-C. Lim, "Robust fault estimation observer in the finite frequency domain for descriptor systems", International Journal of Control, 2017. doi:10.1080/00207179.2017.1403048.

[21]. P. F. Odgaard, J. Stoustrup, M. Kinnaert, "Fault-Tolerant Control of Wind Turbines: A Benchmark Model", IEEE Transactions on control systems technology. Vol. 21, pp. 1168-1182, 2013.

[22]. K. Zhang, B. Jiang, P. Shi, “A new approach to observer-based fault-tolerant controller design for Takagi-Sugeno fuzzy systems with state delay", Circuits Systems \& Signal Processing, Vol. 28, pp. 679-97, 2009.

[23]. P. Gahinet, P. Apkarian, "A linear matrix inequality approach to Ho control", International Journal of Robust and Nonlinear Control. Vol. 4, pp. 421-448, 1994.

[24]. K. Zhang. B. Jiang, V. Cocquempot, "Adaptive Observer-based Fast Fault Estimation", International Journal of Control, Automation, and Systems, Vol. 6, pp. 320-326, 2008.

[25]. V. Gevorgian, M. Singh, E. Muljadi, "Variable frequency operation of a HVDC-VSC interconnected type 1 offshore wind power plant”, 2012 IEEE Power and Energy Society General Meeting, pp. 1-8, 2012.

[26]. M. Rodrigues, H. Hamdi, D. Theilliol, C. Mechmeche, N. BenHadj Braiek, “Actuator fault estimation based adaptive polytopic observer for a class of LPV descriptor systems", Int. J. Robust Nonlinear Control, 2014. DOI: 10.1002/rnc.3236 


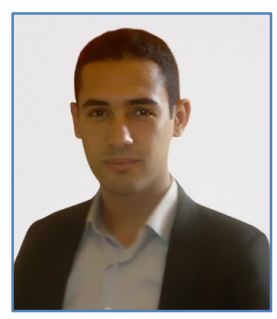

Ayoub EL Bakri received his $\mathrm{PhD}$ degree in physics from the faculty of sciences Dhar el Mehraz at Sidi Mohamed Ben Abdellah University. His research interests include robust control, fault detection and diagnosis, fault-tolerant control, and machine learning with application to nonlinear systems, wind turbines and renewable energy.

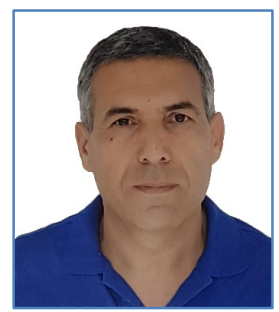

Ismail Boumhidi is a Professor of electronics at the Faculty of Sciences, Fez Morocco. He received from Sidi Mohamed ben Abdellah University, Faculty of sciences his "Doctorat de 3ième cycle" degree in 1994 and "Doctorat d'Etat" degree in 1999. His research areas include adaptive robust control, multivariable nonlinear systems, and fuzzy logic control with applications. 